\title{
Global Stability Analysis for Synchronous Reference Frame Phase-Locked Loops
}

\author{
Zhiyong Dai, Senior member, IEEE, Guangqi Li, Mingdi Fan, Senior member, IEEE, Jin Huang, Yong Yang \\ and Wei Hang
}

\begin{abstract}
This paper analyzes the global stability of synchronous reference frame phase-locked loops (SRF-PLLs), from a large signal viewpoint. First, a large signal model of SRFPLL is accurately established, without applying any linearization method. Then, According to the phase portrait tool, the global stability of SRF-PLL is discussed in the nonlinear frame. Compared with existing methods, the proposed analysis, not relying on small signal model and linearization method, provides a global discussion of SRF-PLL stability. Some novel discoveries are as follow: 1) SRF-PLL has infinite equilibrium points, including stable points and saddle points; 2) Although saddle points are unstable in local regions, there still exists two special lines for each saddle point, and SRF-PLL converges to a certain saddle point when the initial states are on its special lines. 3) These special lines of saddle points divide the global region of SRF-PLL into infinite small regions. A Lyapunov-based discussion proves that SRF-PLL converges to different stable points, when the initial states are in the different small regions. The experiment results have been verified the global stability analysis of SRF-PLL.
\end{abstract}

Index Terms-Grid synchronization, Phase-locked loop, Synchronous reference frame, Global stability analysis, Large signal model.

\section{INTRODUCTION}

With the increasing penetration of renewable energy sources, such as wind power and solar power, a large number of power electronic devices are connected into grids [1]. The grid synchronization is one of the most important technologies for power electronic converters to achieve connection with grids [2]. Literature [3], [4] provide many grid synchronization methods such as SOGI-PLLs [5]-[7], TD-PLLs [8], adaptive observers [9] and etc.. Synchronous reference frame phaselocked loop (SRF-PLL) is a classical grid synchronization method and has been widely used in many kinds of grid-tied converters.

SRF-PLL estimates the phase and the frequency of the grid voltage and it provides the information to achieve grid synchronization of grid-tied converters. The structure of SRFPLL is shown in Fig. 1, and it contains three parts including the phase detector (PD), the loop filter (LF) and the voltagecontrolled oscillator (VCO) [10]. In details, the output of the PD contains the phase error information. LF is a PI controller

This work was supported in part by the National Young Natural Science Foundation of China under Grants 51907137 and 51977136.

${ }^{1}$ Z. Dai, G. Li and J. Huang are with the School of Mechano-Electronic Engineering, Xidian University, Xi'an 710071, China.

${ }^{2}$ M. Fan and Y. Yang are with the School of Rail Transportation, Soochow University, Suzhou, 215131, China.

${ }^{3} \mathrm{~W}$. Hang is with College of Mechanical Engineering, Zhejiang University of Technology, Hangzhou 310023, China.

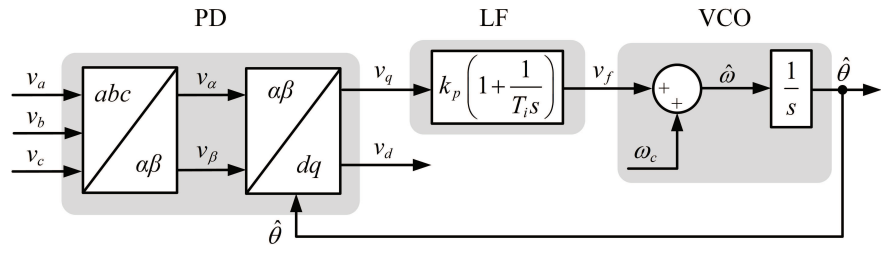

Fig. 1: Structure of SRF-PLL

and it attenuate the high-frequency components from the PD output. VCO generates the estimated phase, which follows the actual phase of the grid voltage.

Recently, it is found that SRF-PLL causes an important affect on the stability of power grids, and several papers have discussed the relationship between SRF-PLL and grid stability [11]-[16]. Generally, the existing methods simplify SRF-PLL as a small signal model [10] and the linearization process is as follows.

When the grid is ideal, the grid voltages $v_{a}, v_{b}$ and $v_{c}$ are

$$
\begin{aligned}
& v_{a}=V \cos (\theta)=V \cos (\omega t+\phi) \\
& v_{b}=V \cos \left(\theta-\frac{2 \pi}{3}\right)=V \cos \left(\omega t+\phi-\frac{2 \pi}{3}\right) \\
& v_{c}=V \cos \left(\theta+\frac{2 \pi}{3}\right)=V \cos \left(\omega t+\phi+\frac{2 \pi}{3}\right)
\end{aligned}
$$

where $V, \omega$ and $\theta$ are the amplitude, frequency and phase angle, respectively. Applying the $a b c-d q$ transformation

$$
T=\frac{2}{3}\left[\begin{array}{ccc}
\cos (\hat{\theta}) & \cos \left(\hat{\theta}-\frac{2 \pi}{3}\right) & \cos \left(\hat{\theta}+\frac{2 \pi}{3}\right) \\
-\sin (\hat{\theta}) & -\sin \left(\hat{\theta}-\frac{2 \pi}{3}\right) & -\sin \left(\hat{\theta}+\frac{2 \pi}{3}\right)
\end{array}\right]
$$

where $\hat{\theta}$ is the estimation of $\theta$. the grid voltages (1)-(3) are deduced as

$$
\begin{aligned}
& v_{d}=V \cos (\theta-\hat{\theta}) \\
& v_{q}=V \sin (\theta-\hat{\theta})
\end{aligned}
$$

(6) is strong nonlinear. To simplify the model of SRF-PLL, a linearization condition is provided as

(A1): the initial estimation error of the phase angle $\theta-\hat{\theta}$ is sufficient small.

With this assumption, (6) is linearized as

$$
\sin (\theta-\hat{\theta}) \approx \theta-\hat{\theta}
$$

From (6)-(7), it is carried out

$$
v_{q}=V \sin (\theta-\hat{\theta}) \approx V(\theta-\hat{\theta})
$$


Hence, when satisfying the assumption (A1), the SPF-PLL in Fig. 1 is linearized as shown in Fig. 2. And the small-signal model of the SRF-PLL is established as [10]

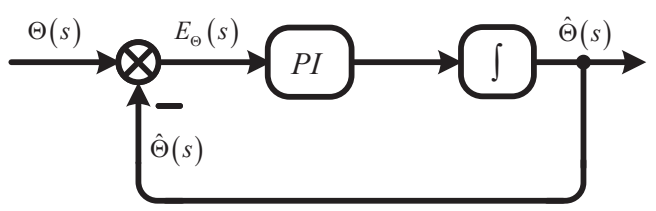

Fig. 2: Small-signal model of SRF-PLL

$$
E_{\theta}(s)=\frac{s^{2}}{s^{2}+k_{p} s+k_{i}}
$$

where $k_{p}$ and $k_{i}$ are the parameters of PI.

According to the small signal model such as (9), the existing methods discuss the SRF-PLL performance. However, these methods are limited by the linearization condition (A1), and the analysis of SRF-PLL is only a local result. To fill this gap, a global analysis of SRF-PLL is provided in this paper. First, a large signal model is established according to the structure of SRF-PLL, which does not rely on the linearization. Then, the theoretical analysis shows the stability of SRF-PLL, globally. Experiment results further confirm the proposed theoretical analysis. The main contributions of this paper are as follows;

1) It is found that SRF-PLL exists infinite equilibrium points that are located at $(n \pi, 0), n=0, \pm 1, \pm 2, \cdots$. In them, $(2 k \pi, 0)$ are stable points and $((2 k+1) \pi, 0)$ are saddle points, where $k=0, \pm 1, \pm 2, \cdots$. When the states are on stable points or saddle points, the states of SRF-PLL will remain at this equilibrium points for all future time.

2) In the local region around a saddle point, SRF-PLL is unstable. However, there still exists two special lines for each saddle point, and SRF-PLL converges to a certain saddle point when the initial states are on its special lines.

3) These special lines of saddle points divide the global region of SRF-PLL into infinite small regions. When the initial states are in the different small regions, SRF-PLL converges to different stable points.

\section{PRELIMINARIES}

In this section, the related nonlinear system theory is introduced, including the definitions of phase portraits, equilibrium points, stable points and saddle points.

Consider a second-order nonlinear system

$$
\begin{aligned}
& \dot{x}_{1}=f_{1}\left(x_{1}, x_{2}\right) \\
& \dot{x}_{2}=f_{2}\left(x_{1}, x_{2}\right)
\end{aligned}
$$

Let $x(t)=\left(x_{1}(t), x_{2}(t)\right)$ be the solution of (10) that starts at a certain initial state $x(0)=\left(x_{1}(0), x_{2}(0)\right)$. The locus in the $x_{1}-x_{2}$ plane of the solution $x(t)$ for all $t \geq 0$ is a curve that passes through the point $x(0)$. The $x_{1}-x_{2}$ plane is usually called the phase plane. The family of all solution curves in $x_{1}-x_{2}$ plane is called the phase portrait of (10).
A point $x=x^{*}$ is said to be an equilibrium point of (10) if it has the property that whenever the state of the system starts at $x^{*}$, it will remain at $x^{*}$ for all future time. The equilibrium points of (10) are the real roots of the equation

$$
\begin{aligned}
& f_{1}\left(x_{1}, x_{2}\right)=0 \\
& f_{2}\left(x_{1}, x_{2}\right)=0
\end{aligned}
$$

Let $p=\left(p_{1}, p_{2}\right)$ be an equilibrium point of (10). It is defined that $y_{1}=x_{1}-p_{1}$ and $y_{2}=x_{2}-p_{2}$. Under a sufficiently small neighborhood the equilibrium point, the nonlinear system (10) is linearized as

$$
\left(\begin{array}{l}
\dot{y}_{1} \\
\dot{y}_{2}
\end{array}\right)=A\left(\begin{array}{l}
y_{1} \\
y_{2}
\end{array}\right)
$$

where

$$
A=\left.\left[\begin{array}{ll}
\frac{\partial f_{1}}{\partial x_{1}} & \frac{\partial f_{1}}{\partial x_{2}} \\
\frac{\partial f_{2}}{\partial x_{1}} & \frac{\partial f_{2}}{\partial x_{2}}
\end{array}\right]\right|_{x=p}
$$

When all the eigenvalues of the matrix $A$ have negative real part, the equilibrium point $p$ is called stable point. When the matrix $A$ has a eigenvalue with positive real part, and a eigenvalue with negative real part, the equilibrium point $p$ is called saddle point.

\section{LARGE-SIGNAL MODEL FOR SRF-PLL}

Small signal model is based on the linearization method and hence it only describes the performace of SRF-PLL in a local region. To analyze SRF-PLL in the global region, a large signal model is established as follows.

According to the LF and VCO parts in SRF-PLL, it is deduced that

$$
\begin{aligned}
\hat{\theta} & =\int \hat{\omega} d s \\
\hat{\omega} & =k_{p} v_{q}+k_{i} \int v_{q} d s+\omega_{c}
\end{aligned}
$$

where $\omega_{c}$ is a constant value. It implies that

$$
\begin{aligned}
& \dot{\hat{\theta}}=\hat{\omega}, \\
& \dot{\hat{\omega}}=k_{p} \dot{v}_{q}+k_{i} v_{q}
\end{aligned}
$$

Defining $\tilde{\theta}=\hat{\theta}-\theta$ and $\tilde{\omega}=\hat{\omega}-\omega$, and noting

$$
\dot{\theta}=\omega, \quad \dot{\omega}=0,
$$

it yields

$$
\begin{aligned}
& \dot{\tilde{\theta}}=\hat{\omega}-\omega=\tilde{\omega} \\
& \dot{\tilde{\omega}}=k_{p} \dot{v}_{q}+k_{i} v_{q}
\end{aligned}
$$

From (6), it is deduced

$$
\dot{v}_{q}=-V \cos \tilde{\theta} \dot{\tilde{\theta}}=-V \tilde{\omega} \cos \tilde{\theta}
$$

Submitting (6) and (18) into (17) leads to

$$
\begin{aligned}
\dot{\tilde{\theta}} & =\tilde{\omega} \\
\dot{\tilde{\omega}} & =-k_{i} V \sin \tilde{\theta}-k_{p} V \tilde{\omega} \cos \tilde{\theta}
\end{aligned}
$$

This is the large-signal model for SRF-PLL. Compared with small signal model, the proposed large-signal model, not relying on linearization and (A1), describes the performance of SRF-PLL, globally. Observing (19), the large model of SRFPLL is strong nonlinear. In the next section, the global stability of SRF-PLL is analyzed based on the proposed model (19). 


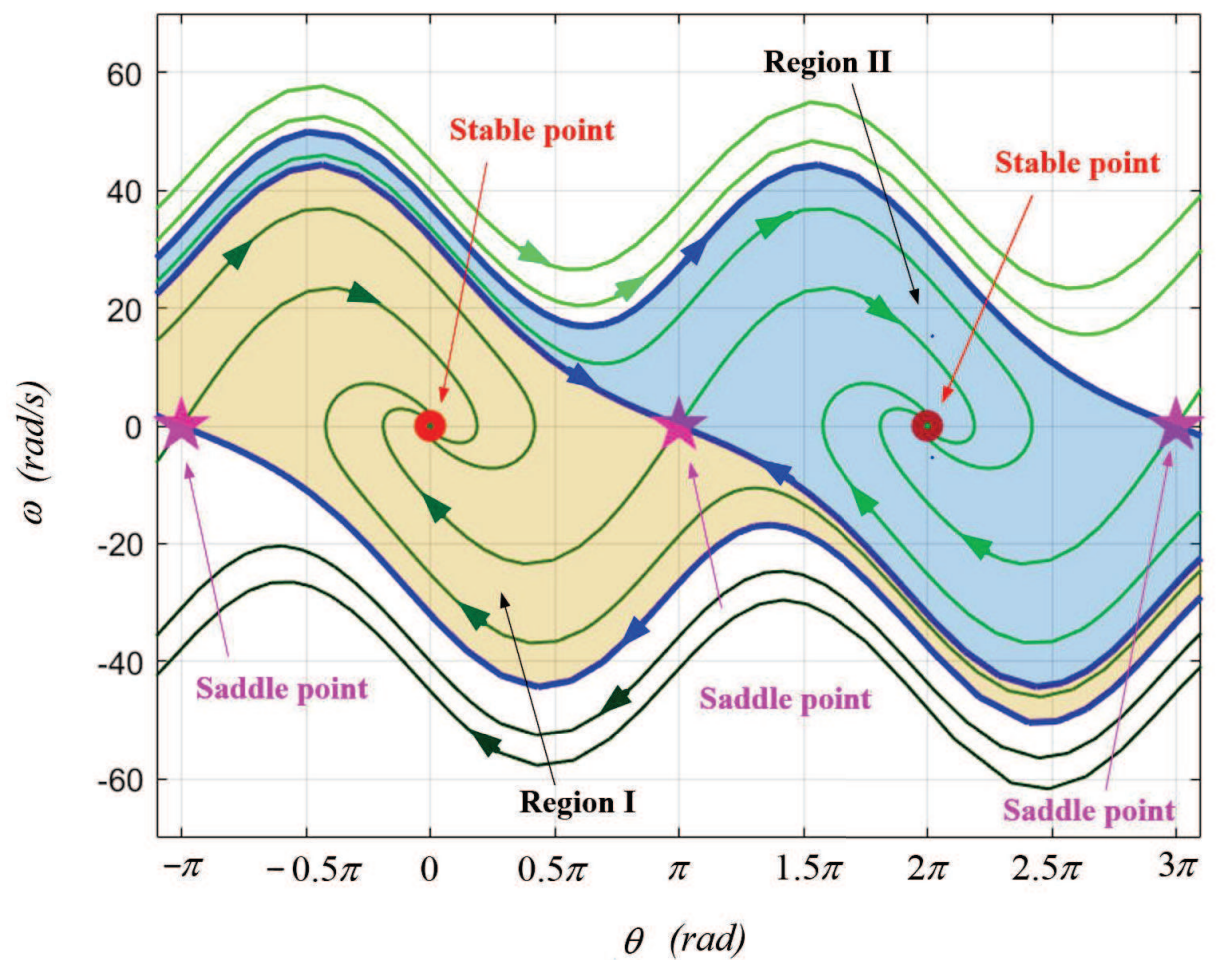

Fig. 3: Phase portrait of SRF-PLL.

\section{LARGE-SIGNAL ANALYSIS}

For nonlinear systems, the phase portrait is a power tool to analyze stability, globally. In this section, the stability of SRF-PLL is discussed according to the phase portrait analysis. Based on the large signal model (19), the phase portrait of SRF-PLL is in Fig. 3. And the step-by-step way is provided in Appendix to draw the Fig. 3. The phase-portrait-based conclusion are as follows.

\section{A. Infinite equilibrium points of SRF-PLL}

According to the definition of equilibrium points shown in Section II, the equilibrium points for the SPF-PLL (19) are the real roots of the equation

$$
\begin{aligned}
& 0=\tilde{\omega} \\
& 0=-k_{i} V \sin \tilde{\theta}-k_{p} V \tilde{\omega} \cos \tilde{\theta}
\end{aligned}
$$

Solving the algebraic equations (20) implies the SPF-PLL has infinite equilibrium points that are located at $(n \pi, 0), n=$ $0, \pm 1, \pm 2, \cdots$.

In what follows, an analysis is provided to show the qualitative behavior near an equilibrium points $(n \pi, 0)$. This is determined via linearization with respect to that point. Firstly, it is defined $\tilde{\theta}_{n}=\tilde{\theta}-n \pi$ and $\tilde{\omega}_{n}=\tilde{\omega}-0$. Then, by the linearization steps in Section II, under a sufficiently small neighborhood of the equilibrium points $(n \pi, 0)$, the nonlinear state equation (19) is approximated by the linear state equations

$$
\left(\begin{array}{c}
\dot{\tilde{\theta}}_{n} \\
\dot{\tilde{\omega}}_{n}
\end{array}\right)=A_{n}\left(\begin{array}{c}
\tilde{\theta}_{n} \\
\tilde{\omega}_{n}
\end{array}\right)
$$

where

$$
A_{n}:=\left[\begin{array}{cc}
0 & 1 \\
-k_{i} V \cos (n \pi) & -k_{p} V \cos (n \pi)
\end{array}\right]
$$

If $n=2 k \pi, k=0, \pm 1, \pm 2, \cdots$, it yields $\cos (n \pi)=1$. Thus, a direct calculation implies the matrix $A_{n}$ has two eigenvalues with negative real part. This means that the equilibrium points $(2 k \pi, 0), k=0, \pm 1, \pm 2, \cdots$, are stable and they are called stable points. In Fig. 3 , Points $(0,0)$ and $(2 \pi, 0)$ are stable points.

Moreover, if $n=(2 k+1) \pi, k=0, \pm 1, \pm 2, \cdots$, the matrix $A_{n}$ has a eigenvalue with positive real part, and the other with negative real part. Thus, these equilibrium points $((2 k+$ 1) $\pi, 0), k=0, \pm 1, \pm 2, \cdots$ are unstable, and they are called saddle points. In Fig. 3, Points $(-\pi, 0),(\pi, 0)$ and $(3 \pi, 0)$ are saddle points.

According to the definition of equilibrium points, it concludes that the initial states of SRF-PLL are on stable points or saddle points, its states will remain at this equilibrium points for all future time.

\section{B. Different stable regions of SRF-PLL}

According to the above analysis of saddle points, SRF-PLL in the small region of saddle points is unstable. It implies that the initial states in this region will go far away the saddle points; while there still exists and only exists two special lines around each saddle point. When the initial states are on the special lines, SRF-PLL will converge to the saddle point [17]. In Fig. 3, the blue lines are these special lines. 


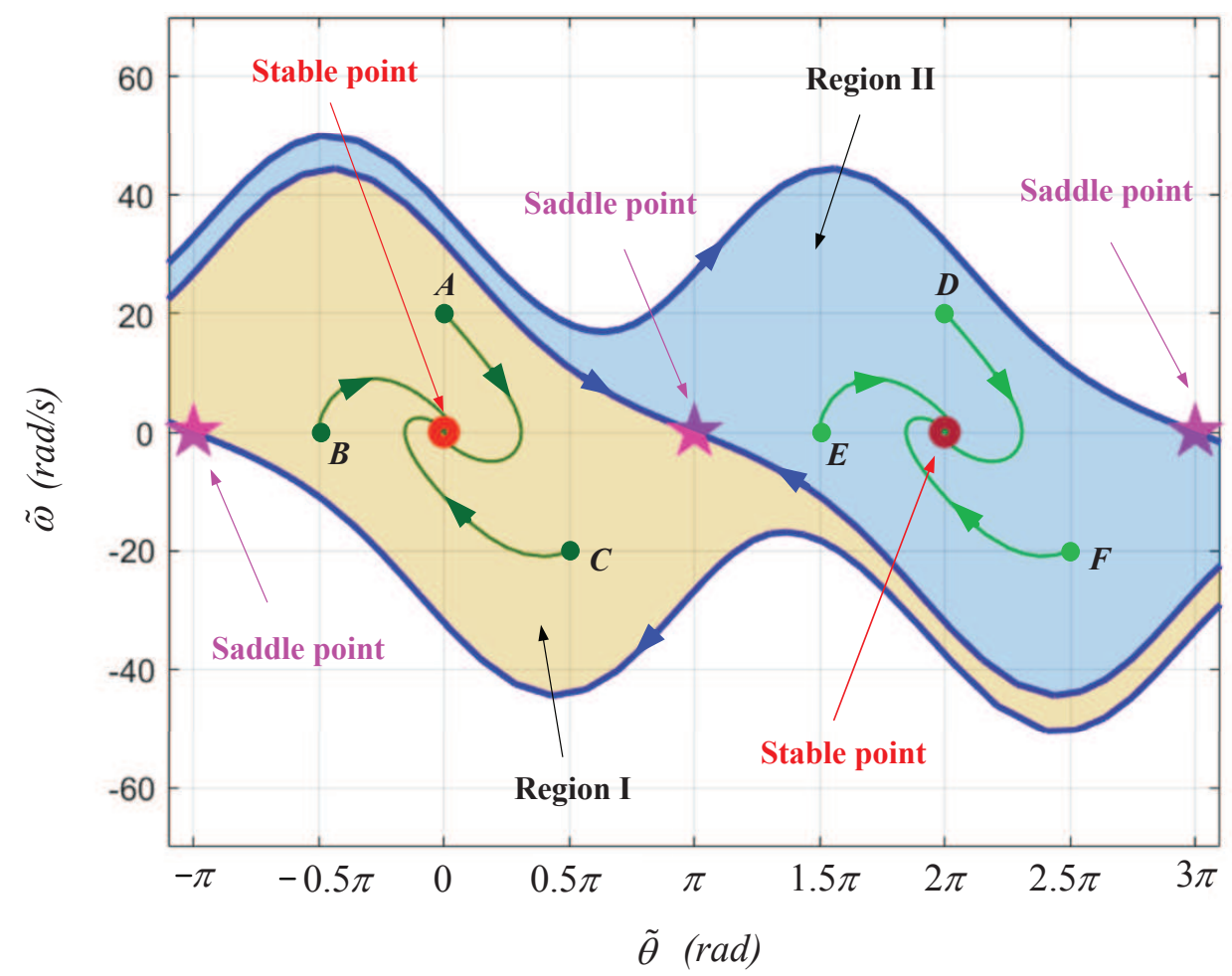

Fig. 4: SRF-PLL Phase portrait with initial states A-F.

Moveover, these special lines divide the global region of SRF-PLL into infinite regions. In Fig. 3, Region I and Region II are highlighted as an example of different regions. When the initial states fall in different regions, SPF-PLL will converge to different stable points $(2 k \pi, 0), k=0, \pm 1, \pm 2, \cdots$, . In a certain region, a large signal proof for SRF-PLL is provided to show the stability as follows.

It is defined the coordination transformation

$$
\tilde{\theta}_{2 k}=\tilde{\theta}-2 k \pi \quad \text { and } \quad \tilde{\omega}_{2 k}=\tilde{\omega}-0
$$

From (19), it is expressed as

$$
\begin{aligned}
& \dot{\tilde{\theta}}_{2 k}=\tilde{\omega}_{2 k} \\
& \dot{\tilde{\omega}}_{2 k}=-k_{i} V \sin \tilde{\theta}_{2 k}-k_{p} V \tilde{\omega}_{2 k} \cos \tilde{\theta}_{2 k}
\end{aligned}
$$

Consider the Lyapunov function

$$
V\left(\tilde{\theta}_{2 k}, \tilde{\omega}_{2 k}\right)=\int_{0}^{\tilde{\theta}_{2 k}} k_{i} V \sin (y) d y+\frac{1}{2} \tilde{\omega}_{2 k}^{2}
$$

It is easy to verify $V\left(\tilde{\theta}_{2 k}, \tilde{\omega}_{2 k}\right)$ is positive in the compact set $\Omega=\left\{\left(\tilde{\theta}_{2 k}, \tilde{\omega}_{2 k}\right) \in \mathrm{R}^{2} \mid V\left(\tilde{\theta}_{2 k}, \tilde{\omega}_{2 k}\right) \leq k_{i} V\right\}$.

From (23) and (24), it is deduced that

$$
\begin{aligned}
\dot{V}\left(\tilde{\theta}_{2 k}, \tilde{\omega}_{2 k}\right)= & k_{i} V \sin \left(\tilde{\theta}_{2 k}\right) \tilde{\omega}_{2 k}-k_{i} V \sin \left(\tilde{\theta}_{2 k}\right) \tilde{\omega}_{2 k} \\
& -k_{p} V \cos \left(\tilde{\theta}_{2 k}\right) \tilde{\omega}_{2 k}^{2} \\
= & -k_{p} V \cos \left(\tilde{\theta}_{2 k}\right) \tilde{\omega}_{2 k}^{2} \leq 0
\end{aligned}
$$

Hence, (25) is negative semidefinite and SRF-PLL in this region is stable.

In the following, the steady-state errors of SRF-PLL is analyzed by LaSalles theorem. Let $S=\{x \in \Omega \mid \dot{V}=0\}$.
Note that

$$
\dot{V}=0 \Rightarrow k_{p} V \cos \left(\tilde{\theta}_{2 k}\right) \tilde{\omega}_{2 k}^{2} \Rightarrow \tilde{\omega}_{2 k}=0
$$

Hence, $S=\left\{x \in \Omega \mid \tilde{\omega}_{2 k}=0\right\}$. Let $\left(\tilde{\theta}_{2 k}(t), \tilde{\omega}_{2 k}(t)\right)$ be a solution that belongs identically to $S$ :

$$
\tilde{\omega}_{2 k} \equiv 0 \Rightarrow \dot{\tilde{\omega}}_{2 k} \equiv 0 \Rightarrow k_{i} V \sin \left(\tilde{\theta}_{2 k}\right) \equiv 0
$$

It results in $\tilde{\theta}_{2 k} \equiv 0$. Therefore, the only solution that can stay identically in $S$ is the trivial solution $\left(\tilde{\theta}_{2 k}(t), \tilde{\omega}_{2 k}(t)\right) \equiv 0$. According to the LaSalle's theorem [17], it concludes that for any initial states $\left(\tilde{\theta}_{2 k}(0), \tilde{\omega}_{2 k}(0)\right) \in \Omega$, the solutions $\left(\tilde{\theta}_{2 k}(t), \tilde{\omega}_{2 k}(t)\right)$ approaches 0 as $t \rightarrow \infty$. Hence, the estimations of SRF-PLL are converged to the stable points $(2 k \pi, 0), k=0, \pm 1, \pm 2, \cdots$, as

$$
\begin{aligned}
\lim _{t \rightarrow \infty} \hat{\theta}_{2 k}(t) & =2 k \pi \\
\left.\lim _{t \rightarrow \infty} \hat{\omega}_{2 k}(t)\right) & =0
\end{aligned}
$$

From the above analysis, the global region of SRF-PLL is divided into infinite stable regions. The initial states in different regions result in SRF-PLL convergence to different stable points $(2 k \pi, 0), k=0, \pm 1, \pm 2, \cdots$.

\section{EXPERIMENT RESULTS}

In this section, SRF-PLL is implemented on a $D S P 28335$ based platform and the above analysis is verified by the following experiment results. The parameters of SRF-PLL is selected as $k_{p}=92$ and $k_{i}=4232$. The initial states of SRF-PLL are choosing as Case A-H shown in Tab. I. The experiment results are shown in Fig. 4 - Fig. 8, respectively. 


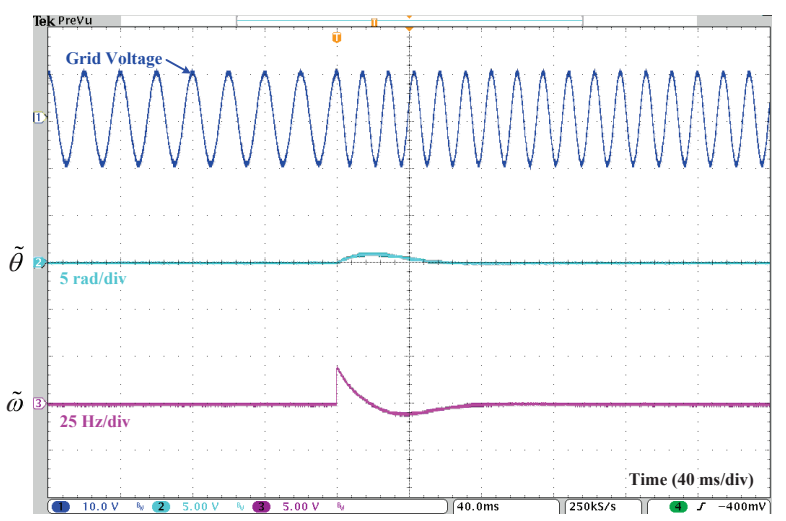

(a) Initial state in Case $\mathrm{A}$

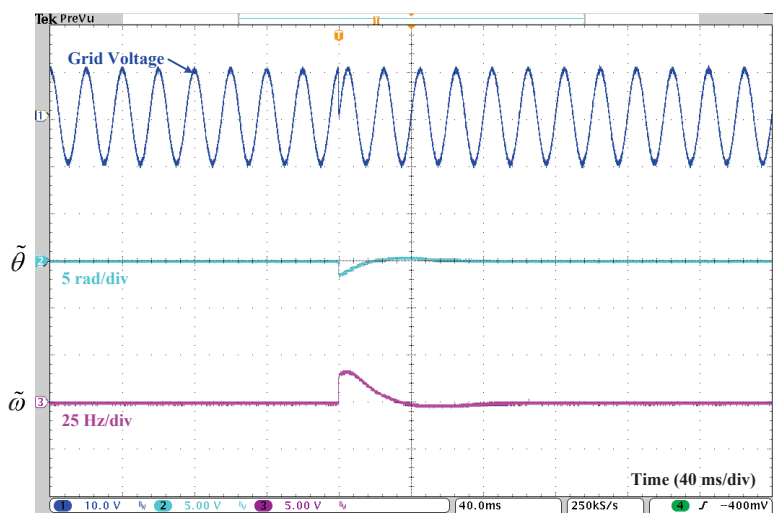

(b) Initial state in Case B

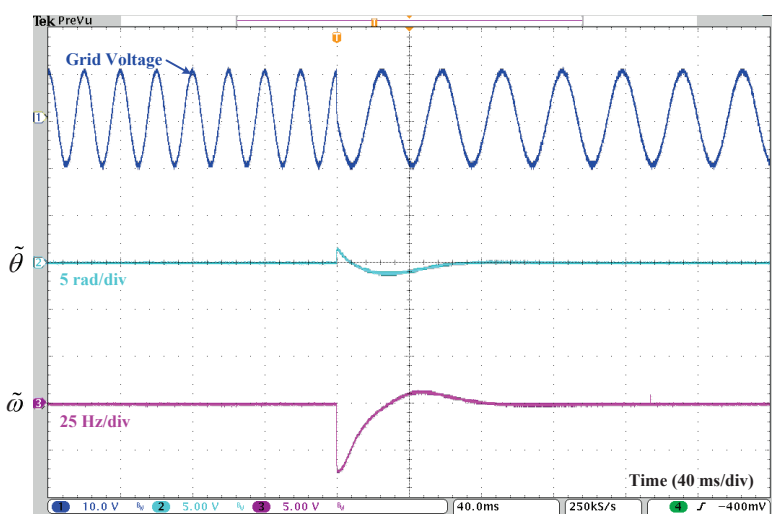

(c) Initial state in Case $\mathrm{C}$

Fig. 5: SRF-PLL performance comparisons with different initial states: Region I.

TABLE I: Different initial states in experimental methods

\begin{tabular}{c|l}
\hline \hline Cases & Initial states (Phase and frequency jump) \\
\hline Case A & $\tilde{\theta}(0)=0 \mathrm{rad}, \tilde{\omega}(0))=20 \mathrm{~Hz}$ \\
Case B & $\tilde{\theta}(0)=-0.5 \pi \mathrm{rad}, \tilde{\omega}(0))=0 \mathrm{~Hz}$ \\
Case C & $\tilde{\theta}(0)=0.5 \pi \mathrm{rad}, \tilde{\omega}(0))=-20 \mathrm{~Hz}$ \\
Case D & $\tilde{\theta}(0)=2 \pi \mathrm{rad}, \tilde{\omega}(0))=20 \mathrm{~Hz}$ \\
Case E & $\tilde{\theta}(0)=1.5 \pi \mathrm{rad}, \tilde{\omega}(0))=0 \mathrm{~Hz}$ \\
Case F & $\tilde{\theta}(0)=2.5 \pi \mathrm{rad}, \tilde{\omega}(0))=-20 \mathrm{~Hz}$ \\
Case G & $\tilde{\theta}(0)=\pi-0.01 \mathrm{rad}, \tilde{\omega}(0))=0 \mathrm{~Hz}$ \\
Case H & $\tilde{\theta}(0)=\pi+0.01 \mathrm{rad}, \tilde{\omega}(0))=0 \mathrm{~Hz}$ \\
\hline \hline
\end{tabular}

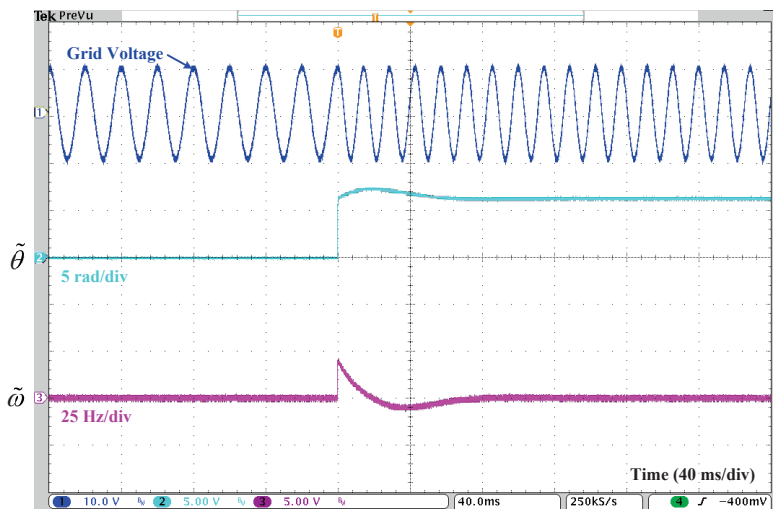

(a) Initial state in Case D

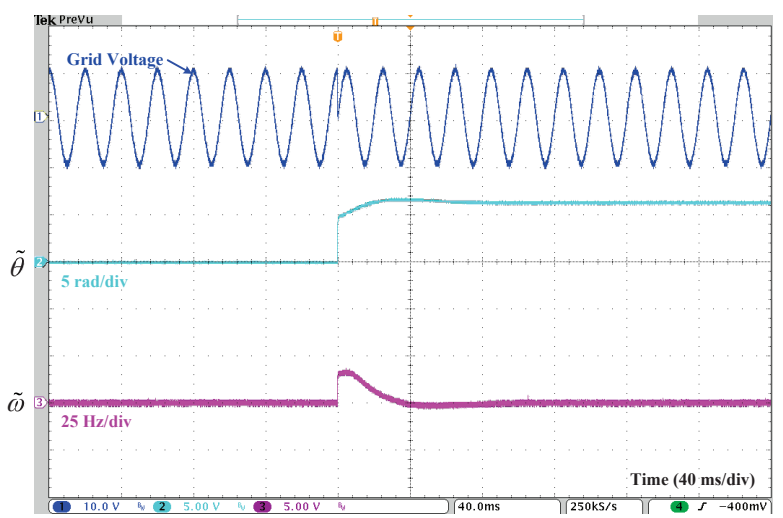

(b) Initial state in Case $\mathrm{E}$

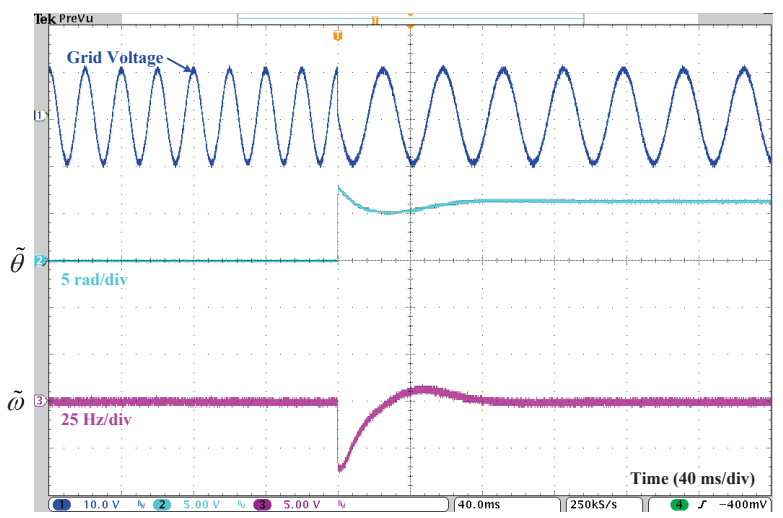

(c) Initial state in Case F

Fig. 6: SRF-PLL performance comparisons with different initial states: Region II.

Fig. 4, Fig. 5 and Fig. 6 provide SRF-PLL performance comparisons with different initial states. Fig. 4 is the phase portraits of SRF-PLL, with the initial states in Case A - Case F. Fig. 5 and Fig. 6 are the estimation responses of SRF-PLL, correspondingly. Case A and Case $\mathrm{D}$ are $20 \mathrm{~Hz}$ frequency jumps. Case B and Case E are $-0.5 \pi$ rad phase jumps. Case $\mathrm{C}$ and Case $\mathrm{F}$ are $-20 \mathrm{~Hz}$ frequency jumps with $0.5 \pi \mathrm{rad}$ phase jumps. Case A - Case $\mathrm{C}$ are in the same region, namely Region I, and they converge to the same stable point $(0,0)$; while Case D - Case F are in the other same region, i.e., Region II, and the steady-states of Case D - Case F go to the same stable point $(2 \pi, 0)$.

Fig. 7 and Fig. 8 show the phase portrait and the estimation 


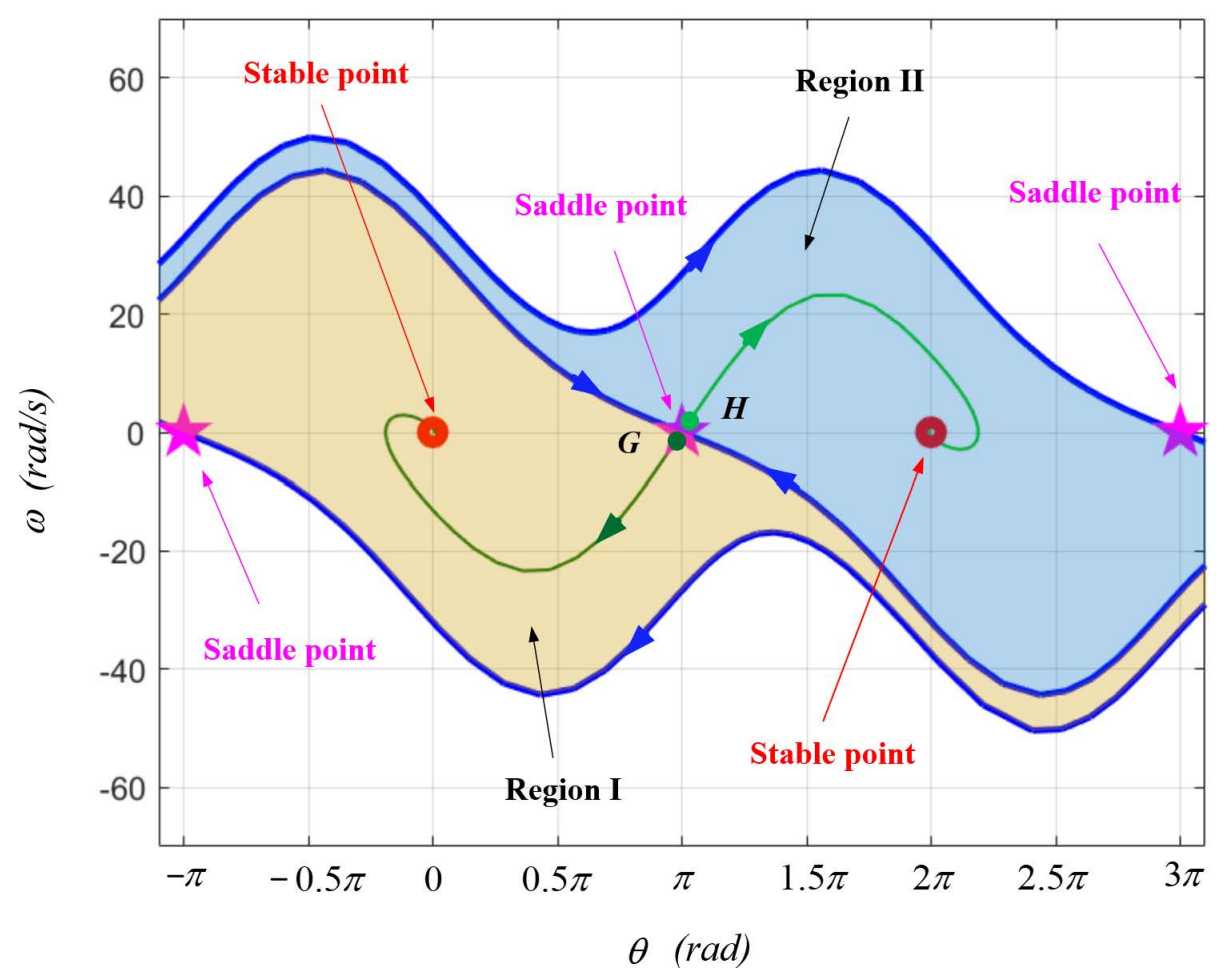

Fig. 7: SRF-PLL Phase portrait with initial states G-H.

response of Case $\mathrm{G}$ and Case $\mathrm{H}$. The initial states of Case $\mathrm{G}$ and Case $\mathrm{H}$ are closed each other. However, they are in the different stable regions, respectively. And it results in that SRF-PLL in Case $\mathrm{G}$ and Case $\mathrm{H}$ converge to stable points $(0,0)$ and $(2 \pi, 0)$, respectively.

Remark: According to theoretical part, the states of SRFPLL will remain at the saddle points for all future time when the states first stand on its saddle point. Meanwhile, there exists two special lines for each saddle point and SRF will converge to the saddle points when its initial states are on these special lines. However, due to the truncation errors of the experiment platform, the initial states of SRF-PLL are difficult to exactly stand on saddle points or special lines, and its experiments are hard to provided in practical cases.

\section{Vi. Conclusions}

Many literature provide the stability discussion of SRF-PLL relying on small signal model and linearization. Hence, it is a local result for SRF-PLL stability. Unlike existing methods, this paper globally analyzes the stability of SRF-PLL, in a nonlinear frame. First, a large signal model of SRF-PLL is accurately established, without linearization. Then, a large signal model-based theoretical analysis is proposed to discuss the stability of SRF-PLL, globally. It is found that SRF-PLL has infinite equilibrium points, including stable points and saddle points. When the states of SRF-PLL stand on stable points or saddle points, its states will stay at this equilibrium points for all future time. Meanwhile, In the local region around a saddle point, SRF-PLL is unstable. It is noted that there exists two special lines for one saddle point, and SRFPLL will converge to the saddle point when the initial states are on these special lines. The special lines of saddle points are plotted by the tool of phase portrait. Furthermore, these special lines divide the global region of SRF-PLL into infinite small regions. When the initial states are in the different small regions, SRF-PLL will converge to different stable points.

\section{APPENDIX}

In this section, the step-by-step way is provided to draw the phase portraits of SRF-PLL.

Step 1. For the large-signal model (19) of SRF-PLL, find all equilibrium points and determine the type of isolated ones via linearization. From above analysis, the equilibrium points $(2 k \pi, 0), k=0, \pm 1, \pm 2, \cdots$, are stable points, and the equilibrium points $((2 k+1) \pi, 0), k=0, \pm 1, \pm 2, \cdots$ are saddle points.

Step 2. Select a bounding box in the state plane and select initial points inside the bounding box. That is, choose different initial values $(\tilde{\theta}(0), \tilde{\omega}(0))^{T}$ such that

$$
\tilde{\theta}_{\text {min }} \leq \tilde{\theta}(0) \leq \tilde{\theta}_{\text {max }}, \quad \tilde{\omega}_{\text {min }} \leq \tilde{\omega}(0) \leq \tilde{\omega}_{\text {max }}
$$

Step 3. For each initial values, calculate the trajectories using numerical algorithm. To find the trajectory passing through a point $(\tilde{\theta}(0), \tilde{\omega}(0))^{T}$, solve the equation

$$
\begin{aligned}
& \dot{\tilde{\theta}}=\tilde{\omega} \\
& \dot{\tilde{\omega}}=-k_{i} V \sin \tilde{\theta}-k_{p} V \tilde{\omega} \cos \tilde{\theta}
\end{aligned}
$$

in forward time (with positive $t$ ) and in reverse time (with negative $t$ ). Solution in reverse time is equivalent to solution in forward time of the equation

$$
\begin{aligned}
\dot{\tilde{\theta}} & =-\tilde{\omega} \\
\dot{\tilde{\omega}} & =k_{i} V \sin \tilde{\theta}+k_{p} V \tilde{\omega} \cos \tilde{\theta}
\end{aligned}
$$




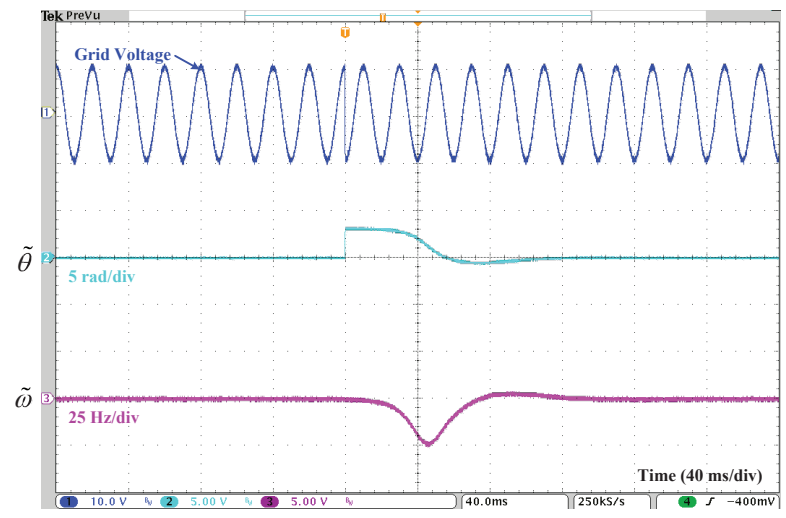

(a) Initial state in Case $\mathrm{G}$

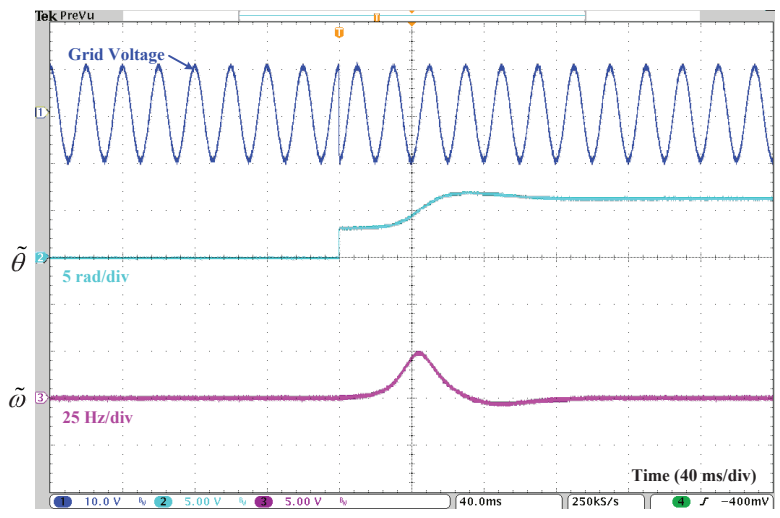

(b) Initial state in Case $\mathrm{H}$

Fig. 8: SRF-PLL performance comparisons with different initial states.

Step 4. For saddle points $((2 k+1) \pi, 0), k=0, \pm 1, \pm 2, \cdots$, use linearization to generate the stable and unstable trajectories. Specifically, let the eigenvalues of the matrix $A_{2 k+1}$ be $\lambda_{1}>0>\lambda_{2}$ and the corresponding eigenvectors be $v_{1}$ and $v_{2}$. The unstable trajectories are drawn by solving the equation in forward time

$$
\begin{aligned}
& \dot{\tilde{\theta}}=\tilde{\omega} \\
& \dot{\tilde{\omega}}=-k_{i} V \sin \tilde{\theta}-k_{p} V \tilde{\omega} \cos \tilde{\theta} \\
& (\tilde{\theta}(0), \tilde{\omega}(0))^{T}=((2 k+1) \pi, 0)^{T} \pm \alpha v_{1}
\end{aligned}
$$

where $\alpha$ is a small positive number. The stable trajectories are drawn by solving the equation in backward time

$$
\begin{aligned}
& \dot{\tilde{\theta}}=\tilde{\omega} \\
& \dot{\tilde{\omega}}=-k_{i} V \sin \tilde{\theta}-k_{p} V \tilde{\omega} \cos \tilde{\theta} \\
& (\tilde{\theta}(0), \tilde{\omega}(0))^{T}=((2 k+1) \pi, 0)^{T} \pm \alpha v_{2}
\end{aligned}
$$

That is, solve the equation in forward time

$$
\begin{aligned}
& \dot{\tilde{\theta}}=-\tilde{\omega} \\
& \dot{\tilde{\omega}}=k_{i} V \sin \tilde{\theta}+k_{p} V \tilde{\omega} \cos \tilde{\theta} \\
& (\tilde{\theta}(0), \tilde{\omega}(0))^{T}=((2 k+1) \pi, 0)^{T} \pm \alpha v_{2}
\end{aligned}
$$

\section{REFERENCES}

[1] F. Blaabjerg, Y. Yang, D. Yang, and X. Wang, "Distributed powergeneration systems and protection," Proceedings of the IEEE, vol. 105, no. 7, pp. 1311-1331, 2017.

[2] X. Wang and F. Blaabjerg, "Harmonic stability in power electronic-based power systems: Concept, modeling, and analysis," IEEE Transactions on Smart Grid, vol. 10, no. 3, pp. 2858-2870, 2019.

[3] S. Golestan, J. M. Guerrero, and J. C. Vasquez, "Single-phase plls: A review of recent advances," IEEE Transactions on Power Electronics, vol. 32, no. 12, pp. 9013-9030, 2017.

[4] S. Golestan, J. M. Guerrero, and J. C. Vasquez, "Three-phase plls: A review of recent advances," IEEE Transactions on Power Electronics, vol. 32, no. 3, pp. 1894-1907, 2017.

[5] F. Xiao, L. Dong, L. Li, and X. Liao, "A frequency-fixed sogi-based pll for single-phase grid-connected converters," IEEE Transactions on Power Electronics, vol. 32, no. 3, pp. 1713-1719, 2017.

[6] S. Golestan, S. Y. Mousazadeh, J. M. Guerrero, and J. C. Vasquez, "A critical examination of frequency-fixed second-order generalized integrator-based phase-locked loops," IEEE Transactions on Power Electronics, vol. 32, no. 9, pp. 6666-6672, 2017.

[7] Y. Han, M. Luo, X. Zhao, J. M. Guerrero, and L. Xu, "Comparative performance evaluation of orthogonal-signal-generators-based singlephase pll algorithmsa survey," IEEE Transactions on Power Electronics, vol. 31, no. 5, pp. 3932-3944, 2016.
[8] Z. Dai, Z. Zhang, Y. Yang, F. Blaabjerg, Y. Huangfu, and J. Zhang, "A fixed-length transfer delay based adaptive frequency-locked loop for single-phase systems," IEEE Transactions on Power Electronics, vol. 34, no. 5, pp. 4000-4004, 2019.

[9] Z. Dai and W. Lin, "Adaptive estimation of three-phase grid voltage parameters under unbalanced faults and harmonic disturbances," IEEE Transactions on Power Electronics, vol. 32, no. 7, pp. 5613-5627, 2017.

[10] R. Teodorescu, M. Liserre, and P. RodriGuez, Grid Converters for Photovoltaic and Wind Power Systems. Hoboken, NJ, USA: Wiley, 2011.

[11] Z. Shuai, Y. Li, W. Wu, C. Tu, A. Luo, and Z. J. Shen, "Divided dq small-signal model: A new perspective for the stability analysis of threephase grid-tied inverters," IEEE Transactions on Industrial Electronics, vol. 66, no. 8, pp. 6493-6504, 2019.

[12] H. Liu and X. Xie, "Comparative studies on the impedance models of vsc-based renewable generators for ssi stability analysis," IEEE Transactions on Energy Conversion, vol. 34, no. 3, pp. 1442-1453, 2019.

[13] X. Li and H. Lin, "A design method of phase-locked loop for gridconnected converters considering the influence of current loops in weak grid," IEEE Journal of Emerging and Selected Topics in Power Electronics, vol. 8, no. 3, pp. 2420-2429, 2020.

[14] S. Zhou, X. Zou, D. Zhu, L. Tong, Y. Zhao, Y. Kang, and X. Yuan, "An improved design of current controller for lcl-type grid-connected converter to reduce negative effect of pll in weak grid," IEEE Journal of Emerging and Selected Topics in Power Electronics, vol. 6, no. 2, pp. 648-663, 2018.

[15] H. Liu, X. Xie, and W. Liu, "An oscillatory stability criterion based on the unified $d q$-frame impedance network model for power systems with high-penetration renewables," IEEE Transactions on Power Systems, vol. 33, no. 3, pp. 3472-3485, 2018.

[16] Y. He, H. S. Chung, C. Lai, X. Zhang, and W. Wu, "Active cancelation of equivalent grid impedance for improving stability and injected power quality of grid-connected inverter under variable grid condition," IEEE Transactions on Power Electronics, vol. 33, no. 11, pp. 9387-9398, 2018.

[17] H. K. Khalil and J. Grizzle, Nonlinear systems. Upper Saddle River: Prentice Hall, third ed., 2002. 